\title{
Merging microfluidics and sonochemistry: towards greener and more efficient micro-sono-reactors $\dagger \ddagger$
}

\author{
David Fernandez Rivas, ${ }^{* a}$ Pedro Cintas $^{b}$ and Han J. G. E. Gardeniers ${ }^{a}$ \\ Received 31st May 2012, Accepted 29th August 2012 \\ DOI: $10.1039 / \mathrm{c} 2 \mathrm{cc} 33920 \mathrm{j}$

\begin{abstract}
Microfluidics enable the manipulation of chemical reactions using very small amounts of fluid, in channels with dimensions of tens to hundreds of micrometers; so-called microstructured devices, from which the iconic image of chips emerges. The immediate attraction of microfluidics lies in its greenness: use of small quantities of reagents and solvents, and hence less waste, a precise control of reaction conditions, integration of functionality for process intensification, safer and often faster protocols, reliable scale-up, and possibility of performing multiphase reactions. Among the limitations found in microfluidics the facile formation of precipitating products should be highlighted, and in this context, the search for efficient mass and energy transfers is a must. Such limitations have been partially overcome with the aid of ultrasound in conventional flow systems, and can now be successfully used in microreactors, which provide new capabilities. Novel applications and a better understanding of the physical and chemical aspects of sonochemistry can certainly be achieved by combining microfluidics and ultrasound. We will review this nascent area of research, paying attention to the latest developments and showing future directions, which benefit both from the existing microfluidic technology and sonochemistry itself.
\end{abstract}

${ }^{a}$ Mesoscale Chemical Systems Group, MESA + Research Institute, University of Twente, ME147, PO Box 217, 7500 AE, Enschede,

The Netherlands. E-mail: d.fernandezrivas@utwente.nl; Tel: +31534892594

${ }^{b}$ Department of Organic and Inorganic Chemistry,

Faculty of Sciences-UEX, E-06006 Badajoz, Spain

$\dagger$ This article is dedicated to the late Dr José González-García, a sonochemist colleague and friend.

$\ddagger$ This article is part of the ChemComm 'Mechanochemistry' web themed issue.

\section{Introduction and scope}

A few years ago chemists wondered whether traditional roundbottomed flasks would become replaced by microreactors. ${ }^{1}$ Certainly, the use of microdevices now represents a realistic option that is becoming increasingly exploited in many laboratories. At first sight, the idea of using microfluidic technology in areas such as chemical synthesis seems rather unpractical and goes against the large-volume toolbox required by process research or the pharmaceutical industry. Motivations for the

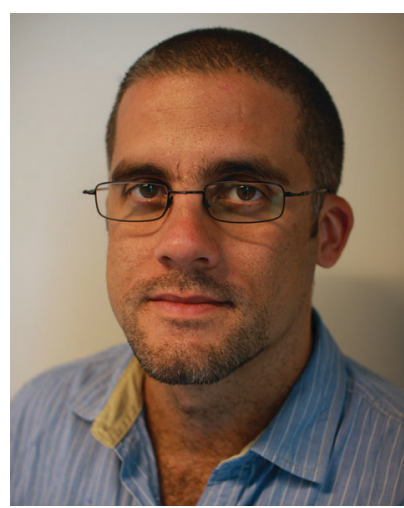

David Fernandez Rivas
David Fernandez Rivas obtained his BSc and MSc in Nuclear Engineering, in 2004 and 2006, respectively, from the Higher Institute of Science and Technology in Havana, Cuba. He pursued his PhD by improving the efficiency of sonochemical reactors using microfluidics and microfabrication techniques under Prof. Gardeniers' supervision at the Mesoscale Chemical Systems of the University of Twente, The Netherlands. His research interests are in the areas of microfluidics, microneedles, solar-to-fuel cells, acoustic cavitation and sonochemistry.

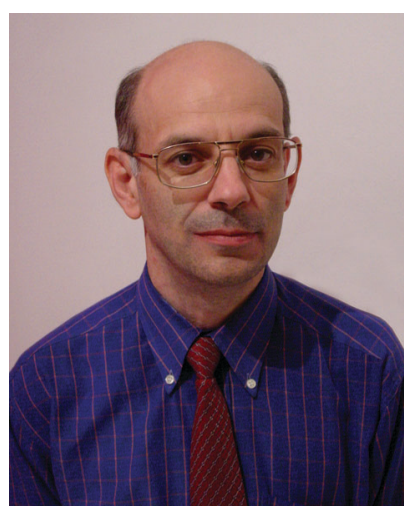

Pedro Cintas
Pedro Cintas has been affiliated with the University of Extremadura (Spain) since 1990, where he is currently professor of organic chemistry. He did postdoctoral research in the University of Geneva (Switzerland, 1989-1990) and also spent time at the University of Paris-Sud (2002) as a visiting professor. His research interests focus largely on green activation methods such as microwave and ultrasound irradiation. He is a member of the advisory board of Ultrasonics Sonochemistry and the scientific committee of the European Society of Sonochemistry. 
development of microfluidics have been, to a significant extent, a triumph of imagination by recognizing that the advantages of microelectronics could be extrapolated to chemical systems, cell biology, and many other fields; ${ }^{2-5}$ and perhaps in part inspired by Feynman's remark: "There is plenty of room at the bottom". ${ }^{6}$ Counter-intuitively, miniaturized reactors have become instrumental in the kilogram-scale production of pharmaceuticals and chemicals involving potentially dangerous steps and more expensive production than for bulk chemicals and requiring additionally a higher research investment per unit weight produced..$^{7-10}$

Although continuous processes based on flow chemistry are also at their height, microreactors comprising small channel sizes and high surface area-to-volume ratios are usually more efficient by several orders of magnitude than their equivalent non-miniaturized counterparts. ${ }^{11}$ In addition, there are fundamental differences between the physical properties of fluids in microchannels relative to those moving through large channels. ${ }^{12-16}$ These physical features will not be detailed in this article, but we highlight a few distinctive characteristics associated with fluid streams in small dimensions. Notably in microfluidics the flow conditions are at low Reynolds numbers (i.e., laminar flow), which indicates that diffusion has a significant role rather than convection; the lack of inertial forces, and the existence of interfacial forces, ${ }^{17}$ and electroosmotic flow, ${ }^{18}$ all of which are ultimately crucial, for instance, in enhanced mixing and particle separations.

There is an ongoing debate as to which dimensions the term microreactor should be employed. ${ }^{12}$ Strictly speaking, the typical dimensions of fluid channels range from the submicrometer to the sub-millimeter level, ${ }^{19}$ with typical low flow rates not exceeding $\sim 1 \mathrm{~mL} \mathrm{~min}^{-1}$. Sometimes, small reactors claimed to involve microstructures having internal volumes of several milliliters, which are markedly different from chip-based reactors. Throughout this article, we will use the term microreactor and reporting channel dimensions whenever available. The puzzling terms nanoreactors, milli- or minireactors have been omitted for the sake of clarity. Microreactors can also be

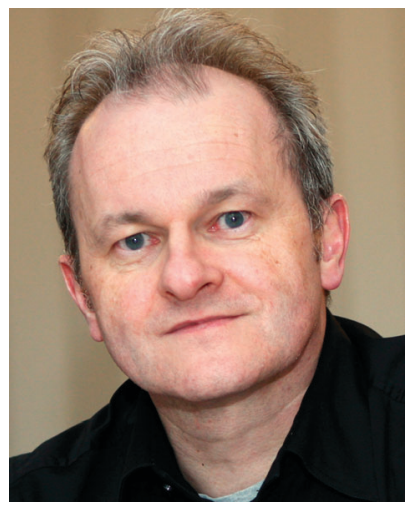

Han Gardeniers (BSc: 1982; MSc: 1985; PhD: 1990) became assistant professor at the University of Twente in 1990. From 2001 till 2003 he was a senior scientist in industry, at Kymata Ltd./Alcatel Optronics and at Micronit Microfluidics. In 2003 he rejoined the University of Twente, where he has been holding a research chair "Mesoscale Chemical Systems", since January 2007. His research interest and expertise is in miniaturized chemical Han J. G. E. Gardeniers synthesis and analysis systems and in nanotechnology related to the study of biomolecular interactions and dynamics in confinement. He has published over 180 reviewed journal papers and is the inventor on 6 patents. classified according to the operation mode, either continuousflow or batch-type. Whereas the former is usually preferred in chemical synthesis, other applications often require batch reactors.

The advent of ultrasound-driven chemical processes, in large or bench scale applications, allows minimisation of problems like fouling and clogging, in continuous operation reactors. Thus, flow reactors benefit from mechanochemical effects such as efficient agitation and mixing after exposure to sound waves. $^{20-23}$ To avoid ambiguities with the use of ultrasound irradiation (to be distinguished from radiological activities), we prefer the term sonication.

Chemical and most physical effects of ultrasound arise from the cavitation collapse of bubbles in a sonicated liquid. Three regions of sonochemistry have been identified: bubble interior, bubble gas-liquid interface and liquid bulk. ${ }^{24-27}$ Suslick and Price have defined three areas of chemical effects of ultrasound: homogeneous sonochemistry of liquids, heterogeneous sonochemistry of liquid-liquid or liquid-solid systems, and sonocatalysis overlaping the first two. ${ }^{20}$ It is worthwhile mentioning that the chemical effects of high-intensity ultrasound are driven by the appearance of bubbles and not due to the interaction of acoustic waves and matter at a molecular or atomic level.

Many sonochemical reactions can be rationalized assuming that volatile substances will undergo homolytic fragmentation and/or thermal excitation inside the bubbles (hot spot theory, which predicts temperatures and pressures in the range of $5000{ }^{\circ} \mathrm{C}$ and $\left.2000 \mathrm{~atm}\right),{ }^{20,24,28,29,102}$ thus producing radical or radical-ion intermediates that can react with species adsorbed at the gas-liquid interface or diffuse into the liquid bulk. ${ }^{30}$ Some other known phenomena are related to the light emission from the interior of the bubbles known as sonoluminescence (SL), either from a single bubble (SBSL) or multibubbles (MBSL). ${ }^{31-33}$ These effects can be understood as particular examples of energy-focusing phenomena.

Bubble collapses are also accompanied by shock waves and shear forces which contribute to mixing and particle fragmentation when solids are present. A plethora of mechanical effects account for enhanced mass and energy transfer, resulting in acceleration, high conversions, rapid emulsification, polymer scission, and even supramolecular organization. ${ }^{24,34-37}$ The mechanical effects that can be pernicious sometimes (e.g. cavitation erosion caused by jetting), when controlled and well understood, can be used in a productive way like in surface cleaning, drug delivery, cell poration, etc. ${ }^{20,38-40}$ Fig. 1 depicts an overview of the multiple phenomena induced by acoustic cavitation.

The combination of microstructures and sonication is a new and emerging area. Such a combination is technically feasible and often leads to synergistic results. Although sonication has been largely employed to generate liquid flow and prevent the deposition of solids, it can do much more. Current research focuses on exploring sonochemical processes themselves with microfluidics. Under such conditions, cavitation collapse and associated phenomena like SL and radical production among others can be optimized and fine-tuned. One should bear in mind that numerous ultrasound-assisted reactions lack reproducibility, partly because cavitational energies depend on 


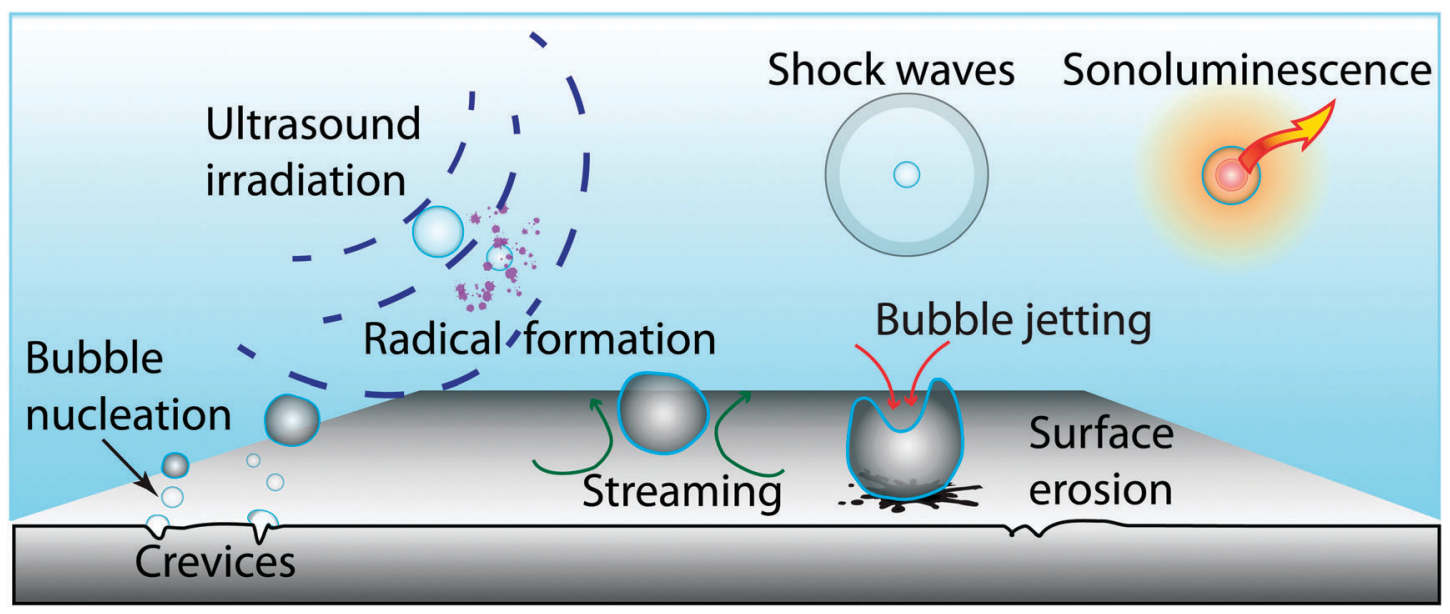

Fig. 1 Schematic representation of the main effects of cavitation induced by ultrasound irradiation (sonication). Original artwork from the authors.

external parameters (frequency, intensity, dissolved gas, etc.) and solvent properties. Microfluidics devices are amenable for a precise control of temperature as well as frequency and pressure amplitudes combined with efficient mixing and short residence times. In addition, microfluidic systems allow the study of reactions at extreme conditions with reduced operation risks (explosive or run-away reactions, etc.). Moreover, large-volume applications only need scaling out or numbering up, which require a design in parallel of multiple reactors. Certain areas of chemical engineering can benefit already from the advantages of microfluidics, mainly those involving the production of pharmaceuticals or fine chemicals. ${ }^{7,10,11,41-43}$

Acoustic energy can be supplied in different forms, as transducers and piezoelectrical microdevices with different sizes and geometries are usually employed. These considerations are especially important regarding sound propagation and attenuation. Sound, unlike electromagnetic radiation, is not quantum energy and therefore parameters other than frequency may have a crucial influence; there is no direct relationship between energy and frequency. Power/intensity and above all fluid properties, will influence the outcome to a larger extent. This overview excludes an in-depth treatment of droplet-based surface acoustic waves (SAWs), which is also a versatile and promising technology, but not directly related to cavitational phenomena in liquids. ${ }^{44}$ The approach of SAWs is based on shock waves with amplitudes of a few nanometers, which are generated by a piezoelectric inter-digital transducer (IDT). Liquid droplets placed on a surface are moved by the acoustic pressure. The technology is often called flat fluidics because no cover or slit is usually required. ${ }^{5} \mathrm{SAW}$ s and other ultrasonic applications, mainly in medicine, use frequencies in the $\mathrm{MHz}$ region, where a high intensity would be required to induce cavitation (in fact $\mathrm{MHz}$ frequencies are employed in medical imaging to avoid tissue damage). ${ }^{21,45-47}$ As we shall see later an important advantage of this miniaturized technology is the facile integration of piezoelectric actuators into microchips, which has been exploited in particle separations. ${ }^{48,49}$ SAWs have also found applications in synthetic chemistry. ${ }^{50}$
It is convenient to highlight that some ultrasound-assisted microstructures do not necessarily involve cavitation, the core of sonochemistry - a fact not always mentioned explicitly in the literature. Therefore, interpretations of putative effects should be assessed with caution, ranging from efficient micromixing to temperature increases, reactor geometries and manufacturers themselves. It is difficult to cover all the current or potential applications of US cavitation in combination with microfluidics, among which we can mention: water treatment, fine chemical synthesis, food industry, sonoelectrochemistry, nanomaterials design and synthesis, drug delivery, dentistry, etc. As this article aims to point out, the combination of both fields can certainly yield new applications and improve the existing ones.

\section{Managing heterogeneous reactions in microreactors}

As mentioned above, the use of acoustic radiation in capillary reactors and chip-based microstructures represents a solution for managing solids and overcomes the limitations of bridging and constriction; thereby enabling the use of high reagent concentrations. A simplified sketch in Fig. 2 illustrates some of these effects.

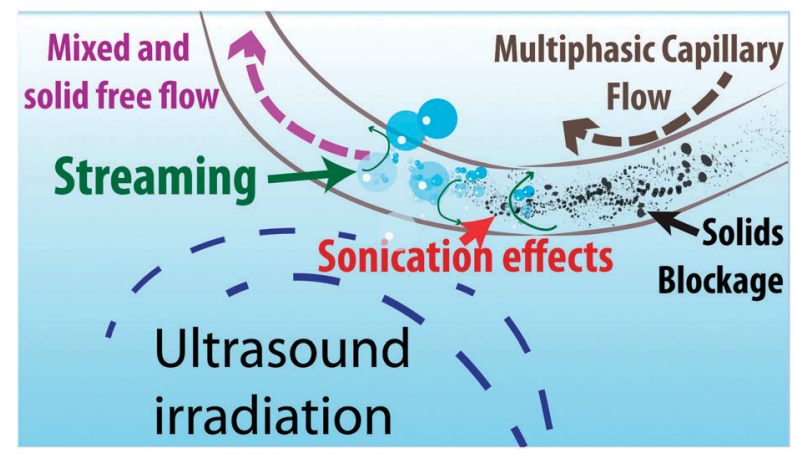

Fig. 2 Schematic representation of sonication effects on a capillary through which a multiphasic (heterogeneous) mixture is pumped. The effects provided either by mixing or cavitation bubbles enhance transport and thereby reaction rates. Original artwork from the authors. 
Some reactors cannot be considered microreactors in the strict sense, as they use a capillary format of millimeter dimensions and pumps that give rise to flow rates in the $\mathrm{mL} \min ^{-1}$ range. Such devices have become commercially available and have certainly revolutionized chemical synthesis. Herein, ultrasound can easily be applied by immersing the loops and tubing into an ultrasonic bath. Recent examples involve the oxidation of alcohols and aldehydes to carboxylic acids, as well as the Nef oxidation of nitroalkenes to carbonyls and acids, which can be conducted in a flow reactor using $\mathrm{KMnO}_{4}$ as an oxidant and pulsed sonication.

Sonication prevents the blocking of the reactor and affords products in high yields and purity. ${ }^{51}$ Buchwald and associates have employed the same strategy for Pd-catalyzed amination reactions in flow. The experimental set-up consisted of syringe pumps to deliver reagents to a microreactor made of polytetrafluoroethylene (PTFE) that was placed in an ultrasonic bath. ${ }^{52,53}$ Likewise, other synthetic transformations have been reported. ${ }^{54}$ The versatility of these ultrasound-based modifications was further illustrated by the photodimerization of maleic anhydride. ${ }^{55}$ The reaction solution was passed through a mm-sized tube, which was simultaneously irradiated with a high-pressure $\mathrm{Hg}$ lamp and an ultrasonic bath to prevent sedimentation and adhesion of solids (Fig. 3).

Biphasic reactions in capillary reactors clearly benefit from emulsification effects provided by sonication, such as in the case of phase-transfer (PT) reactions. ${ }^{56}$ Aqueous and organic fluids are fed by two separate lines and then mixed through a T-shaped connector. Sonication is supplied by a bath where a constant temperature can be maintained by an immersed coilheat exchanger (Fig. 4). In that study, sonication enhanced the conversion due to an efficient agitation that not only improved the mixing within one phase, but also helped in rupturing the interface between both phases, increasing the contact area. This purely mechanical effect could also be corroborated by a combination of mechanical stirring and sonication, which enhanced yields still further.

A model reaction involving the hydrolysis of $p$-nitrophenyl acetate was studied by Wirth et.al. using segmented flow (rather than parallel flow) in a T-shaped geometry. ${ }^{41}$ Fluid segments are stirred by internal vortices generated by the interaction of the liquid with the channel wall. By immersing the microchannel tubing (internal diameter ID $=300 \mu \mathrm{m}$, $L=400 \mathrm{~mm}$ ) in an ultrasonic bath at $20^{\circ} \mathrm{C}$, higher conversions with respect to conventional PT-catalysis and even under

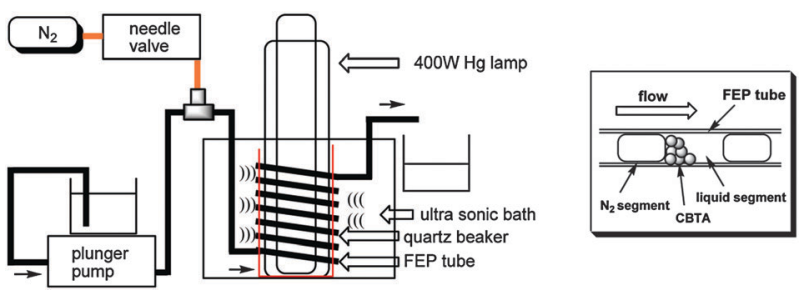

Fig. 3 Clogging averted in a photochemical reaction with the aid of sonication. Inset: magnified microchannel made of fluorinated ethylene propylene (FEP) under flow. CTBA denotes a cyclobutane derivative, the photodimerization product of maleic anhydride. Reprinted with permission from ref. 55. Copyright 2010 American Chemical Society.

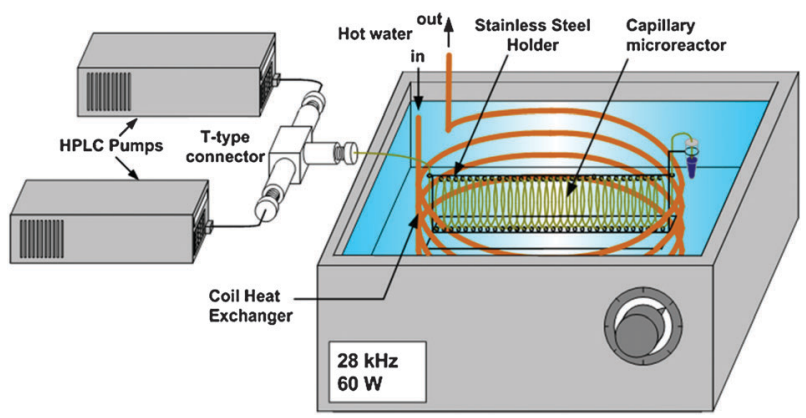

Fig. 4 Schematic representation of an ultrasound-assisted capillary microreactor for the PT catalysis of benzyl chloride with sodium sulfide in the presence of tetrahexylammonium bromide. Reprinted with permission from ref. 56. Copyright 2009 Elsevier B.V.

microwaves at $50{ }^{\circ} \mathrm{C}$ were observed. A slight enhancement could also be obtained by running the reaction with sonication plus a PT-catalyst.

The same hydrolysis has been re-investigated recently, using again segmented flow in a microstructured device (IDs ranging from 300 to $2400 \mu \mathrm{m}$; wall thicknesses of $400-800 \mu \mathrm{m}$ ). Ultrasound was indirectly transmitted to the chemical system through a pressurized vessel filled with water ( $c a .4 .5$ bar). The sonotrode (operating at $20 \mathrm{kHz}$ ) was placed axially at a distance of $c a .35 \mathrm{~mm}$ below the microdevice, which resulted in optimal intensity without causing material damage. The slug flow of the biphasic system could be monitored using a high-speed camera, showing that sonication leads to an apparently complete emulsification, thus increasing the interfacial area. ${ }^{57}$ High yields (up to $86 \%$ ) were obtained with sonication, although they were also influenced by the channel diameter and material. Best results were achieved with PTFE (versus glass and PEEK = polyether ether ketone) tubing. Unfortunately, the set-up did not permit an accurate control of the temperature, especially for large IDs, for which the greatest yield enhancements were observed (with respect to silent conditions).

The above-mentioned design is based on a previous ultrasonic flow-through cell setup, consisting of a cylindrical steel jacket with an inner glass tube (ID $=2 \mathrm{~mm}$ ) for conducting the fluid, which was sonicated by a piezoelectric transducer ( $24 \mathrm{kHz})$. Between the glass tube and the jacket, pressurized water (4.5-5.5 bar) was passed for sound transmission (Fig. 5). ${ }^{43}$ Accordingly the apparatus is well suited for aseptic processing, often required in pharmaceutical and food industries.

Application to rapid formation of vegetable oil-in-water emulsions revealed the influence of both the acoustic power and residence times. Pre-emulsions had oil droplets ranging from 50 to $200 \mu \mathrm{m}$ while ultrasonic processing at $25 \mathrm{~W}$ gave rise to mean diameters of less than $1 \mu \mathrm{m}$, and eventually droplets of 5-10 $\mu \mathrm{m}$. At $32 \mathrm{~W}$, no droplets were microscopically visible and the result was reproducible enough irrespective of the residence time (Fig. 6). Below $17 \mathrm{~W}$ no appreciable emulsification could be observed.

The relationship between the applied pressure and cavitation conditions is very complex and will be affected by the ultrasound frequency and several other parameters (temperature, gas content, etc..${ }^{58}$ Intuitively one would expect that the 


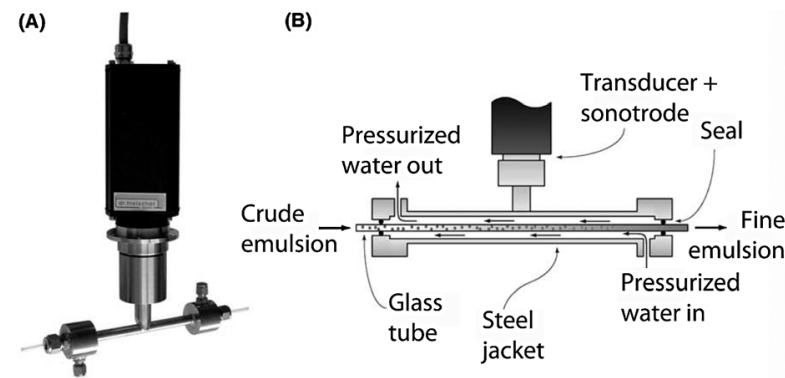

Fig. 5 Ultrasonic flow cell (A: photograph, B: design) in which sound waves $(24 \mathrm{kHz})$ are indirectly transmitted via pressurized water between the steel jacket and the glass tube containing the emulsion. Reproduced with permission from ref. 43. Copyright 2006 Elsevier B.V.
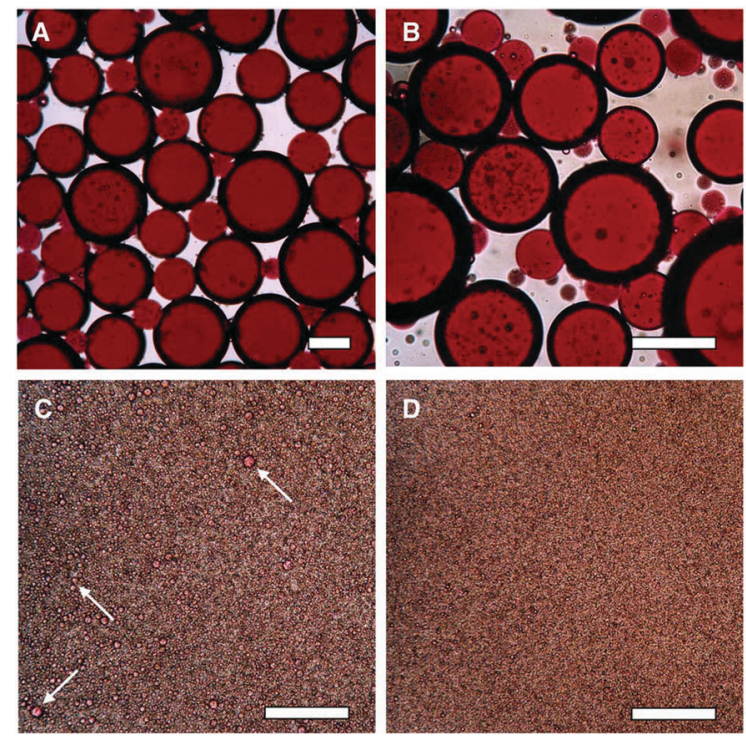

Fig. 6 Light microscope micrographs of pre-mixed emulsions (A: $50 \mu \mathrm{m}$ droplets; B: $200 \mu \mathrm{m}$ droplets) and sonicated emulsions at different acoustic power (C: $25 \mathrm{~W}$; D: $32 \mathrm{~W}$ ) prepared with $20 \%(\mathrm{v} / \mathrm{v})$ olive oil (stained with Fat Red 7B). Residence time: $13 \mathrm{~s}$; bars denote $100 \mu \mathrm{m}$. Arrows in $\mathrm{C}$ show single large droplets $(5-10 \mu \mathrm{m})$. Reproduced with permission from ref. 43. Copyright 2006 Elsevier B.V.

larger the bubble before the collapse, the more energy is available for mechanical or chemical effects. The pressure applied influences the resonance between the acoustic field and the vibration frequency of the bubbles, whose radii are also a function of the pressure. The distance between bubbles and other objects will also have a significant effect on the types of collapses. A complete and thorough explanation is not trivial and has been discussed elsewhere. ${ }^{59-61}$

\section{Miniaturized actuators}

Integration of small piezoelectric transducers into microfluidic channels can be accomplished through a variety of designs. As noted in the introductory remarks, an important application of standing waves in microfluidic chips is the continuous separation of mixed particle suspensions according to their properties in an acoustic field. ${ }^{62,63}$
The interaction of ultrasound with matter is a very complex one, in terms of the lengthscales and timescales involved, material properties, temperature and several other factors. For instance, a standing ultrasonic wave generates stationary pressure gradients, and if in a liquid medium, these gradients exert forces on particles with different density and compressibility; more details on the associated radiation and interparticle forces, heating, etc. are reported in the literature. ${ }^{21,50,64}$

A relatively simple device that showed no significant heating of the fluid was introduced by Yaralioglu et.al. ${ }^{65}$ It consists of a series of transducers made of $\mathrm{ZnO}$ thin films (operating at ca. $450 \mathrm{MHz}$ ) placed at the bottom surface of a quartz substrate. The microfluidic channel made of poly(dimethylsiloxane) (PDMS) is aligned to the transducers on the top surface. The transducers produce acoustic streaming perpendicular to the flow direction, thus enhancing mixing. Flow rates of 1-100 $\mu \mathrm{m} \mathrm{min}^{-1}$ were used for mixtures of phenolphthalein and $\mathrm{NaOH}$ solutions in ethanol. No bubble formation was observed at the $\mathrm{MHz}$-region, which is in contrast to the case when using power ultrasound.

The so-called free flow acoustophoresis enables for instance the fractionation of red cells, platelets and leukocytes using three outlets, provided that the density of the medium is altered with a salt. ${ }^{66}$ The microtransducer worked at $c a .2 \mathrm{MHz}$ and the authors noted that the rise in temperature was less than $10{ }^{\circ} \mathrm{C}$ (Fig. 7).

Acoustic microstreaming using air bubbles in microchannels is also an efficient protocol for micromixing. ${ }^{67}$ The functioning of such a microdevice relies on a piezoelectric disk glued to a microchamber where air bubbles are trapped. The bubble oscillations induce a circulatory flow that results in convection
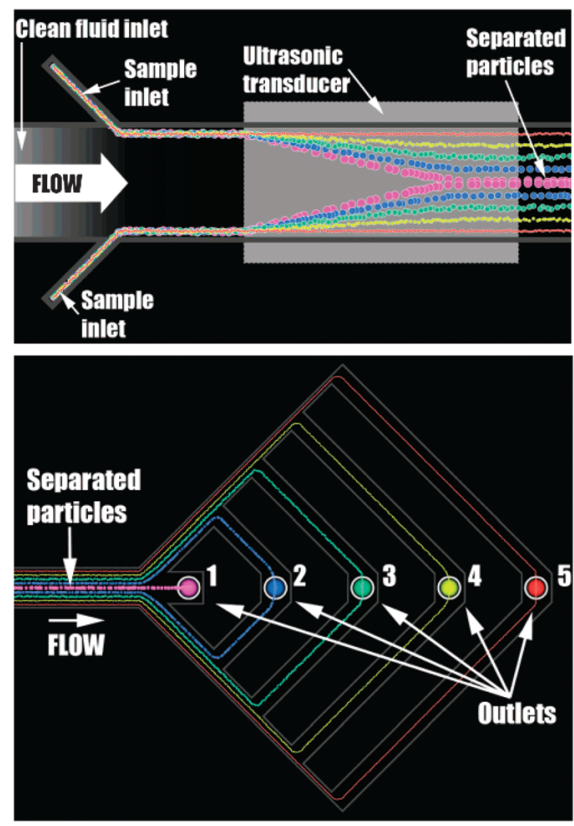

Fig. 7 Scheme showing the action of acoustic standing waves on a particle suspension passing with laminar flow (top). Particles can be fractionated at the end of the channel $(350 \mu \mathrm{m}$ width) through consecutive outlets (bottom). Reproduced with permission from ref. 66. Copyright 2007 American Chemical Society. 


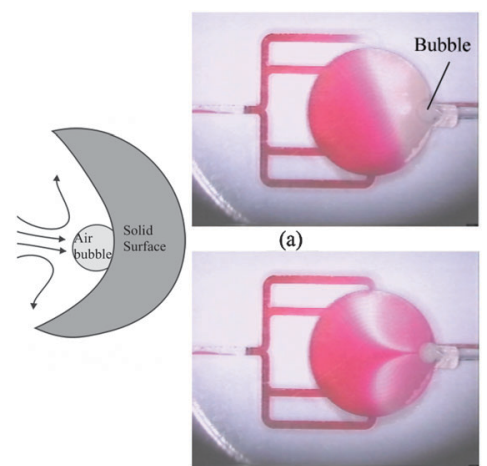

(c)

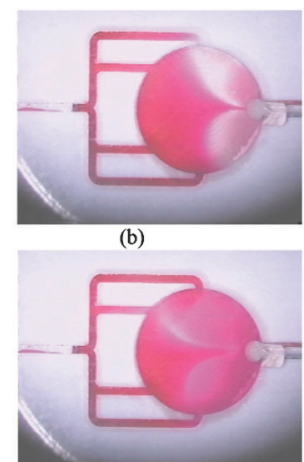

(d)
Fig. 8 Action of acoustic microstreaming induced by an air bubble on a surface (left). Photographs showing microstreaming around a single air bubble in a chamber $(300 \mu \mathrm{m}$ depth, ID $=15 \mathrm{~mm})$ at 0,15 , 35 and $70 \mathrm{~s} \mathrm{(a,} \mathrm{b,} \mathrm{c} \mathrm{and} \mathrm{d,} \mathrm{respectively).} \mathrm{A} \mathrm{piezoelectric} \mathrm{disk} \mathrm{attached} \mathrm{to}$ that chamber is not shown for clarity. Reproduced with permission from ref. 67. Copyright 2002 The Royal Society of Chemistry.

and rapid mixing (Fig. 8). In fact, sonication caused no motion of the liquid if air bubbles were not present, as was visualized with a red dye solution. The bubble-induced streaming is dependent on frequency for a given radius and, conversely on bubble radius for a given frequency. The microsystem worked at $5 \mathrm{kHz}$, below the ultrasonic threshold of $c a .20 \mathrm{kHz}$, although the low voltage also ensured a low power consumption $(c a .2 \mathrm{~mW})$, which makes the apparatus suitable for biological applications as inferred from an E. coli bacterial assay in red cells.

Another example of this type of actuation with intrinsic flow directionality without the need of using microchannels was presented by Marmottant et al. ${ }^{68}$ They used a range of frequencies to make different bubble sizes resonate $(20-200 \mathrm{kHz})$. Doublet arrays featuring pits with radii between 15 and $50 \mu \mathrm{m}$, combined with cylindrical bumps with a radius of $60 \mu \mathrm{m}$ and $30 \mu \mathrm{m}$ height. The distance between the centers of pit and bump within one doublet was of $95 \mu \mathrm{m}$, and the doublets inter-distance $500 \mu \mathrm{m}$. The linear and small oscillations of the bubbles trapped in the pits resulted in vigorous agitation but no cavitation was observed in the liquid. The low ultrasonic amplitudes prevented heating the working fluid. Microfluidic transport was accomplished without pressure gradients and as stated above, without microchannels; making it a good alternative where clogging of channels in two-phase applications is undesired and unavoidable (Fig. 9).

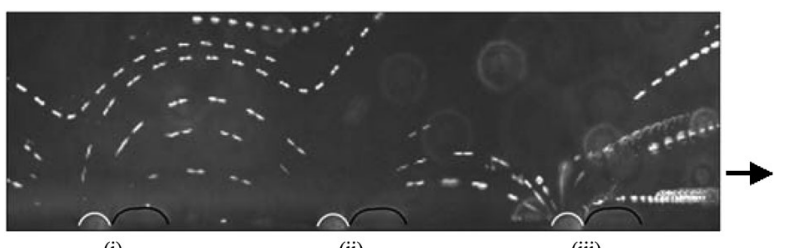

(i)

(ii)

(iii)

Fig. 9 Side view of three bubble/bump doublets in experiment when bubbles are vibrating in response to ultrasound, with long-time exposure showing the transport of fluorescent beads in the indicated direction (bubble radius $a=30 \mu \mathrm{m}$ ). Trajectories in the plane perdicular to the wall are visible. Reproduced with permission from ref. 68. Copyright 2006 Cambridge University Press.
A different design by Huang and associates, also harnesses single-bubble acoustic streaming by trapping an air bubble within a "horse-shoe" microstructure located between two laminar flows in a PDMS-based microchannel $(240 \mu \mathrm{m}$ width, $L=1 \mathrm{~cm})$. A piezoelectric transducer $(70 \mathrm{kHz})$ was bonded to the microfluidic device. Homogenized mixing could be attained within the millisecond range whereas no effect was observed in the absence of sonication. ${ }^{69}$ Related experiments involve the use of oscillating bubbles excited at different $\mathrm{kHz}$-frequencies for particle trapping based on both size and density. ${ }^{70}$

Iida et al. were able to observe microbubble related phenomena, synchronized with the ultrasonic wave by a stroboscopic technique. ${ }^{71}$ Bubble oscillation, diffusion, the onset of cavitation, or microstreaming led to different spatial patterns depending on the input power. Fig. 10 provides a schematic representation of the stroboscopic observation along with the geometry of a microreactor made of PDMS with a microchannel of $200 \mu \mathrm{m}$ width and $50 \mu \mathrm{m}$ depth. A larger microchannel $(400 \mu \mathrm{m}$ width and $200 \mu \mathrm{m}$ depth) was used for microstreaming observations. Sonication was provided via a miniaturized horn-type oscillator $(D=15 \mathrm{~mm}$, tip diameter $=10 \mathrm{~mm}$ ) operating at $c a .60 \mathrm{kHz}$ in contact with the PDMS microreactor. Mechanical effects caused by microstreaming in the above mentioned reactor are also depicted in Fig. 10. Yeast cells were added as tracer particles. On increasing the power density from 0.01 to $0.05 \mathrm{~W} \mathrm{~cm}^{-2}$, the streaming around the bubble increased to the half width of the channel. At $0.10 \mathrm{~W} \mathrm{~cm}{ }^{-2}$ yeast cells moved violently, which may cause cell wall rupture. The direction of microstreaming was not apparently uniform and temporary changes in flow were observed, either toward or from the bubble.

The effect of surface-active solutes on the dynamic behavior of acoustic bubbles (coalescence, clustering, and fragmentation),

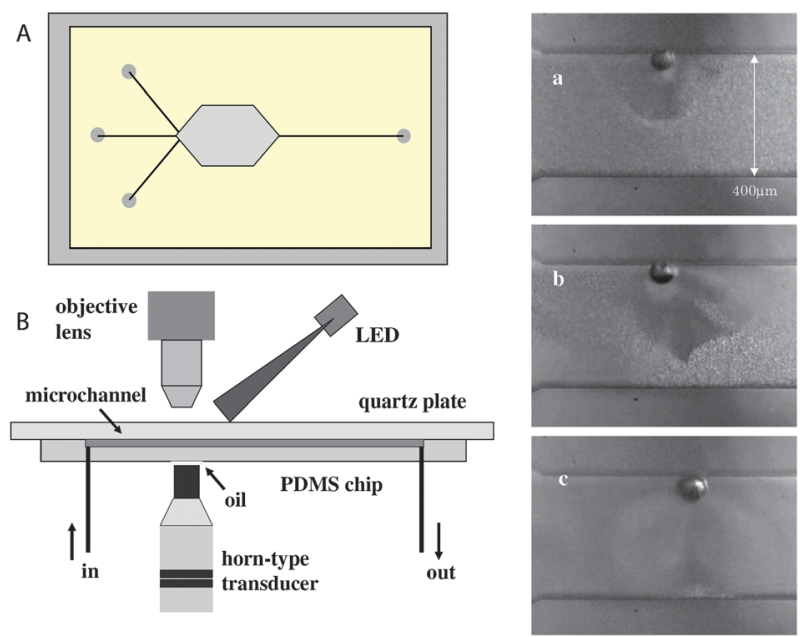

Fig. 10 (A): The microreactor geometry is shown with bold lines and an hexagon representing the channels and pool, respectively. (B): Stroboscopic observation of bubble motions in a micro-sono-reactor. Microstreaming induced by a bubble in a microchannel at different ultrasonic powers: (a) 0.01 , (b) 0.05 and (c) $0.10 \mathrm{~W} \mathrm{~cm}^{-2}$. Tracer particles are yeast cells. Reproduced with permission from ref. 71. Copyright 2007 Elsevier B.V. 
at $60 \mathrm{kHz}$ under microconfinement, was reported by Lee et al. $^{72}$ It was observed that bubbles coalesced instantly in water; however, in the presence of surface-active solutes ( $n$-propanol and sodium dodecyl sulfate, SDS) the coalescence was hindered. The collective oscillation of a bubble cluster consisting of differently sized bubbles and images of bubbles emitting a fountain of microbubbles were reported.

Tandiono and collaborators ${ }^{73}$ performed different studies in PDMS microchannels. A transducer was operated at $100 \mathrm{kHz}$ with pressure and vibration amplitudes of up to 20 bar and $400 \mathrm{~nm}$ measured close to the covering top glass surface. The strong forcing resulted in the excitation of nonlinear surface waves when gas-liquid interfaces are present in the microfluidic channels. Nuclei leading to intense inertial cavitation were generated by the entrapment of gas pockets within the interfaces. Cavitation bubble clusters were recorded with highspeed photography at up to 250000 frames $\mathrm{s}^{-1} .^{73}$

Whereas the above experiments illustrate analytical applications, the synthetic scenario is invariably constrained by solidforming reactions. This concern has so far been solved by combining microfluidic devices with an ultrasonic bath, a strategy that is energetically inefficient with minuses associated with both temperature and power control. Home-made microsono-reactors have been developed by assembling PTFE plates $(70 \times 70 \mathrm{~mm}$, channel width $=600 \mu \mathrm{m})$ with a piezoelectric actuator ( $1 \mathrm{~mm}$ thickness), the latter being driven at different frequencies by a wave generator and amplification to an optimal power of $30 \mathrm{~W}$ (Fig. 11). ${ }^{74}$ The resulting microreactor was used in Pd-catalyzed couplings of anilines and aryl halides, which led to precipitates $(\mathrm{NaCl}$ or $\mathrm{NaBr})$ in apolar solvents. Sonication at $50 \mathrm{kHz}$ prevented the microreactor from clogging (most particles were smaller than $20 \mu \mathrm{m}$ ),

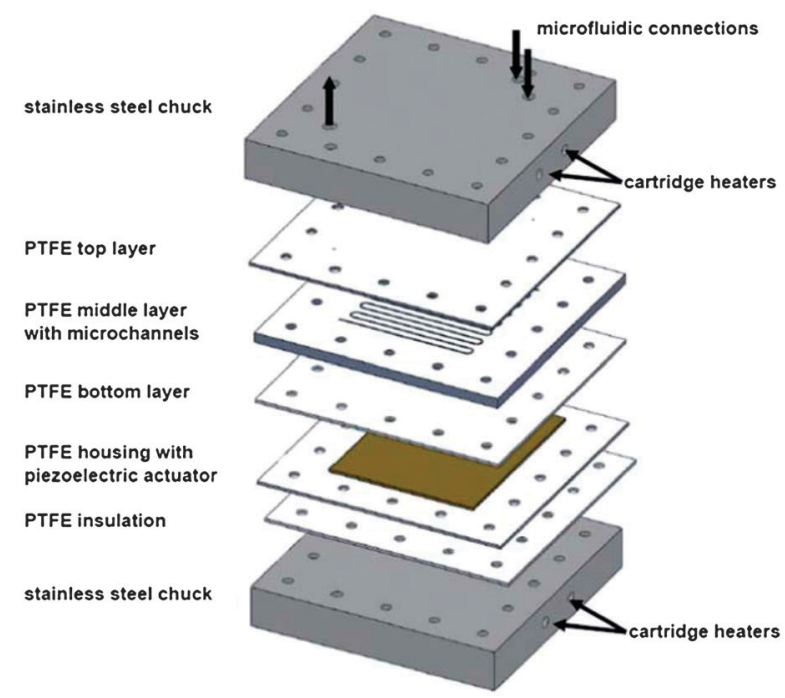

Fig. 11 Representation of a microreactor assembly used for synthetic purposes, which includes three PTFE plates, the middle providing the microchannel structure. A piezoelectric actuator is integrated underneath the pile of PTFE plates and electrically insulated with a Teflon plate. The plates are compressed using two stainless steel chucks with holes for the insertion of cartridge heaters. Reproduced with permission from ref. 74. Copyright 2011 The Royal Society of Chemistry. and excellent yields $(>95 \%)$ could be obtained within 60-90 s. Microchannel monitoring using an inverted microscope indicated that before sonication solid particles were present, which were subsequently broken up after sonication accompanied by bubble formation. This suggests that solvent degassing and cavitation are largely responsible for mechanical effects for that particular experiment.

\section{Sonochemical studies in micro-sono-reactors}

The conditions created after the implosion of cavitation bubbles in a liquid have been shown to enhance numerous chemical reactions ${ }^{30,42,75-77}$ and sometimes with the addition of particles to the liquid ${ }^{78}$ or the modification of the surfaces of a sono-reactor. ${ }^{79}$ Each cavitation bubble can be regarded as a microreactor possessing a high-energy environment (as mentioned before, temperatures of $5000{ }^{\circ} \mathrm{C}$ and pressures of about $2000 \mathrm{~atm}$ ) that produces a plethora of excited chemical species. ${ }^{80,81}$ A single bubble collapsing in a reproducible way could be taken as the idealized reactor; like in experiments of SBSL. ${ }^{82-84}$ Unfortunately, the amount of reagent inside a bubble is so tiny that these conditions have no practical value, though give theoretical support to SL and hot spot theoretical studies. ${ }^{28,85}$ The conditions for the appearance of bubbles and their subsequent cavitation are still not well understood, rendering the reproducibility of a given experiment a challenge defined by several physical-chemical factors. It has been concluded that in any case, each reaction-reactor system has to be optimized to fit a specific application. ${ }^{22,86-90}$

Sonochemists regularly conduct experiments in vessels larger than the active zone where cavitation occurs (either using horns or baths). The problem of matching the ultrasonic field with the reactor dimensions can be partially solved with micromachined surfaces (modified surfaces or microchannels), as cavitation is then confined to small volumes and the nucleation sites for bubble appearance can be better controlled. Controlling cavitation events and a better understanding of such complex systems contributed to an increase in the energy efficiency of an ultrasonic microreactor (sono-micro-reactor) of the bath type. ${ }^{79}$

To our knowledge, the first micro-sono-reactor study was presented by Iida and collaborators, who measured the production of $\mathrm{OH}^{\bullet}$ radicals in microchannels under cavitation. ${ }^{91}$ For comparative purposes, both the cavitational threshold and the efficiency to produce $\mathrm{OH}^{\bullet}$ radicals by water sonolysis (see eqn (1)) were evaluated in 1D- (a microchannel), 2D(a thin layer) and 3D-microreactors, the latter being a glass tube (20 $\mathrm{mm}$ height and $25 \mathrm{~mm}$ diameter).

$$
\mathrm{H}_{2} \mathrm{O} \stackrel{\Delta H=5.1 \mathrm{eV}}{\rightleftharpoons} \mathrm{OH}^{\bullet}+\mathrm{H}^{\bullet}
$$

The corresponding components were tightly glued to a piezoelectric oscillator. The working frequencies were 162, 166 and $158 \mathrm{kHz}$ for 1D, 2D and 3D sono-reactors, respectively. The generation of $\mathrm{OH}^{\bullet}$ radicals produced by cavitation was monitored by their reaction with terephthalate anions giving rise to fluorescent hydroxyterephthalate, which represents a common chemical dosimeter in determining the cavitation threshold (Scheme 1). The detection limit of this dosimeter according to 
<smiles>O=C([O-])c1ccc(C(=O)[O-])cc1</smiles>

Scheme 1 Terephthalate anions as a trap for hydroxy radicals; the corresponding product can be monitored by fluorescence measurements.

the experimental set-up of this study was $2.1 \times 10^{-13} \mathrm{~mol} \mathrm{~mL}^{-1}$ (equivalent to $1.2 \times 10^{11}$ molecules $\mathrm{mL}^{-1}$ ).

The threshold value for the emergence of cavitation in the cases of $1 \mathrm{D}$ and 2D microreactors was ca. $0.7 \mathrm{~W} \mathrm{~cm}^{-2}$, much higher than that determined for the $3 \mathrm{D}$ case $\left(0.02 \mathrm{~W} \mathrm{~cm}^{-2}\right)$. The requirement of a high power density to induce cavitation in confined spaces can be rationalized assuming that bubble dynamics and hence the extension of collapse is constrained by the microspace. This could also account for the fact that cavitation in human tissues requires sound pressures considerably higher than those used in sonochemical reactors. ${ }^{92}$ As expected, these experiments confirmed that the production rates of hydroxyterephthalate anions increased from $1 \mathrm{D}$ to $3 \mathrm{D}$ sono-reactors. However, taking into account the increase in reaction volumes (by 57 times from $2 \mathrm{D}$ to $3 \mathrm{D}$ ), the efficiency of the three-dimensional reactor was poorer (reaction rate increased by 10 times).

Ohl's team, who had done work studying the effect of sonication of PDMS channels, ${ }^{73}$ looked in more detail at SL and sonochemiluminescence (SCL) in the same micro-system. ${ }^{93}$ The bubbles form a planar/pancake shape in the microfluidics channels; and since the chemical reactions are closely confined to gas-liquid interfaces, they concluded that this spatial control of sonochemical reactions would prove useful in lab-on-a-chip devices (see Fig. 12). The chemiluminescent reaction of luminol arises from ultrasonic generation of active radical species after cavitational collapse. Recombination of $\mathrm{OH}^{\bullet}$ radicals produces hydrogen peroxide and this reagent is usually employed in laboratory demonstrations (Scheme 2).

\subsection{Novel concept for micro-sono-reactors}

Further improvements on sonochemical efficiency in microreactors have been reported recently using pits micromachined on a silicon surface. ${ }^{79}$ The application is based on the fundamental idea that, stable and monodisperse cavitation nuclei can be generated by trapping gas in such pits, ${ }^{94}$ which produce high yields at ultrasound powers that otherwise would not lead to a noticeable sonochemical effect. The main advantage of this method is that the location of cavitation occurrence is known a priori, which allows a detailed study of several relevant aspects of sonochemistry, such as SL, SCL and other effects such as erosion, cleaning action of bubbles, shock waves, jetting, streaming, etc.; ${ }^{38,95,96}$ all of which have been difficult to address in the past, given the rather randomness of cavitating bubbles associated phenomena. Though the studies have been carried in the microscale, it has been proposed that this strategy of modifying the walls of a large scale sonoreactor, could lead to improvement in efficiency and versatility of already existent systems.
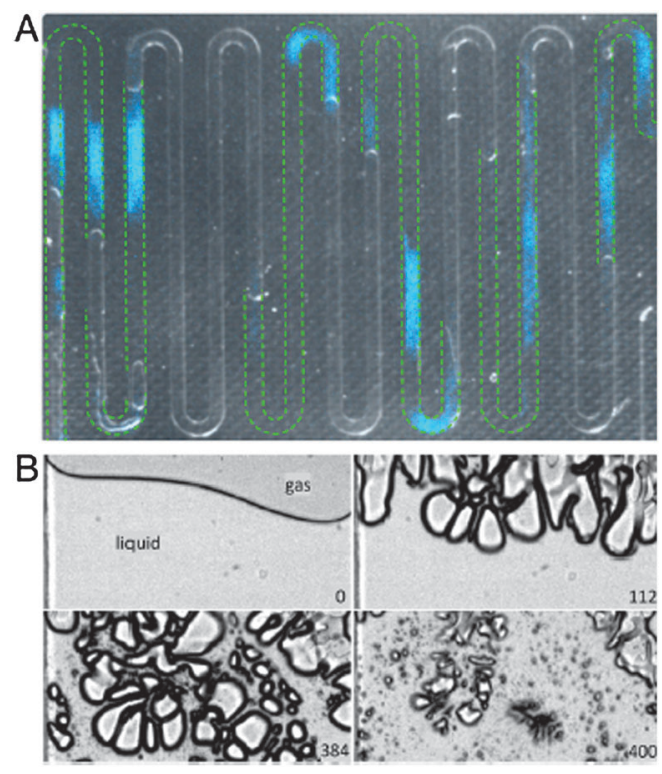

Fig. 12 Luminol chemiluminescence from cavitation bubbles. (A) Composed picture of the channel geometry (gray background) showing the distribution of liquid and gas phases. The green dashed lines indicate the positions of liquid slugs just before the ultrasound is switched on. The bluish patches are taken with an intensified EMCCD camera and capture the chemiluminescence from the oxidation of luminol. (B) Sequence of images taken with a high-speed recording of the bubble distribution near a gas-liquid interface. The number in each frame is the time in microseconds after turning on the ultrasound. They show consecutively the initial gas-liquid interface leading to the severe surface instabilities, large bubble expansions, and finally the collapses. The interface was exposed to a harmonic driving at an amplitude of $230 \mathrm{~V}$ at its resonance frequency of $103.6 \mathrm{kHz}$. The width of each frame (channel) is $500 \mu \mathrm{m}$. Reproduced with permission from ref. 93. Copyright 2007 the American Association for the Advancement of Science (AAAS).

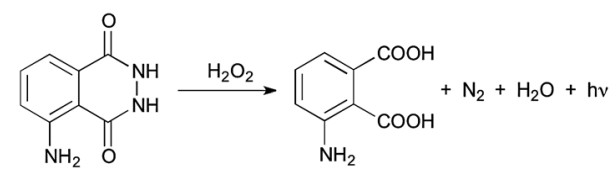

Scheme 2 Free radical species react with luminol producing bright sparks of light in the presence of sonic waves.

The experimental set-up is shown in Fig. 13. The cylindrical pits $(30 \mu \mathrm{m}$ in diameter and depth of $10 \mu \mathrm{m})$ were obtained by using a plasma dry-etching machine and were arranged in three different configurations: single pits, two pits separated by a $1 \mathrm{~mm}$ distance, and three pits at the corners of an equilateral triangle with a side of $1 \mathrm{~mm}$. The pitted silicon chips were placed at the bottom of a glass vessel $(V=300 \mu \mathrm{L})$ in which water sonolysis was investigated at $200 \mathrm{kHz}$ (an ultrasonic transducer was attached to the bottom of the chip).

Three different acoustic powers: $0.074 \mathrm{~W}$ (low), $0.182 \mathrm{~W}$ (medium) and $0.629 \mathrm{~W}$ (high); produce microbubbles ejected from the pits, though their trajectories and patterns are different as a result of Bjerknes forces and the geometry of the micro-sono-reactor as depicted in Fig. 14. A single pit at low and medium powers ejects microbubbles along the axis of 


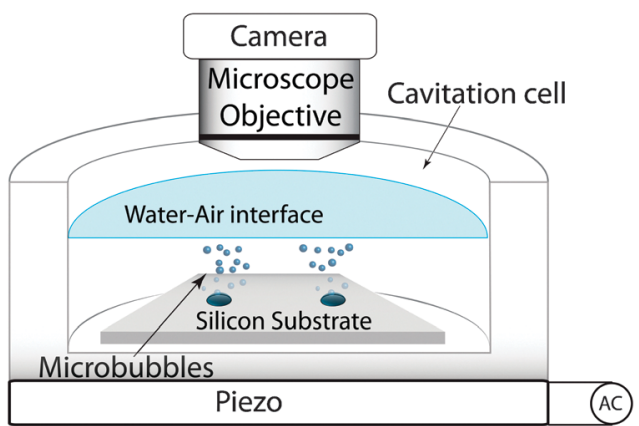

Fig. 13 Experimental set-up showing the components to generate cavitation on pit-containing micromachined silicon substrates. The piezo is controlled by an amplified wave generator signal. This system can provide top and side views of the cavitation events provided by the nucleation of bubbles from artificial crevices etched into a smooth silicon surface.

the pit that follow an ascending trajectory. This trend was also observed for two and three pits at low power. On increasing power, the microbubbles moved away from the symmetry axis of the pit and towards one another, thus creating a dense cloud around the midpoint of the pit arrangement.

It is interesting to compare the power values of this type of reactor (see Table 1) with other reported values. ${ }^{91}$ For the calorimetric values, the low power is comparable $\left(0.7 \mathrm{~W} \mathrm{~cm}^{-2}\right)$, whereas for larger power, this approach of gluing the piezo to the glass container seems to be able to inject more energy into the system $\left(2.59 \mathrm{~W} \mathrm{~cm}^{-2}\right)$.

The evidence of the chemical activity provided by these cavitation bubbles was visualized by SCL of aqueous solutions of luminol (see Fig. 14). Quantitative estimations of $\mathrm{OH}^{\bullet}$
Table 1 Electrical and calorimetric powers and their corresponding density values used in the micropits based sono-reactor

\begin{tabular}{lllll}
\hline Power & $\begin{array}{l}P_{\text {Electr. }} \\
(\mathrm{W})\end{array}$ & $\begin{array}{l}P_{\text {Electr. }} / \text { area } \\
\left(\mathrm{W} \mathrm{cm}^{-2}\right)\end{array}$ & $\begin{array}{l}P_{\text {Calorim. }} \\
(\mathrm{W})\end{array}$ & $\begin{array}{l}P_{\text {Calorim. }} / \text { area } \\
\left(\mathrm{W} \mathrm{cm}^{-2}\right)\end{array}$ \\
\hline Low & 0.074 & 0.047 & 3.32 & 2.11 \\
High & 0.629 & 0.400 & 12.7 & 8.09 \\
\hline
\end{tabular}

radicals by reaction with terephthalate anions clearly proved that the presence of pits enhanced significantly the radical formation rate, which was proportional to the number of pits and power setting. Though, the slope of the reaction rate curve shows a descending trend as the number of pits and power applied increased, which could be attributed to bubble-bubble and bubble cluster-cluster interactions. ${ }^{95,96}$ In contrast, nonpitted silicon substrates showed little evidence of ultrasonic effect. One could figure out that linear extrapolation to multiplepit configurations in a sono-reactor will largely increase its efficiency. The nucleation of microbubble from the pits, and the subsequent cavitation of the gas seeded by the collapse of the initial nucleated bubbles, and perhaps other effects, as enhanced microstreaming, are certainly important. ${ }^{96}$

How the presence of the pits can influence the microsono-reactor flow conditions is shown in Fig. 15 (recorded at 250 frames per second), where the liquid was seeded with gold particles and the superposition of the recorded images allows the visualization of streaklines.

When amplitudes are kept lower than $80 \mathrm{kPa}$ for one pit, there is no cavitation and the bubble trapped on the pit induces the streaming. When bubbles nucleate from the pit at higher amplitudes, the streak lines are changed and an increase in the liquid circulation takes place.

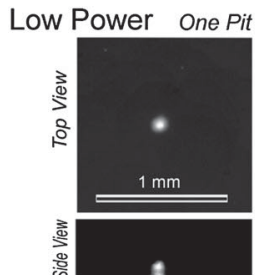

Medium Power
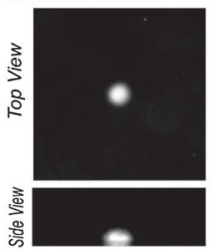

High Power

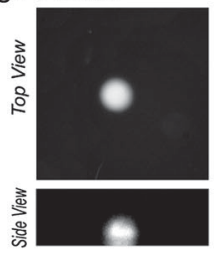

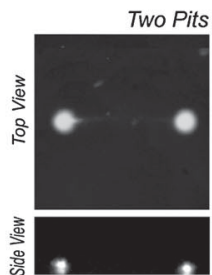
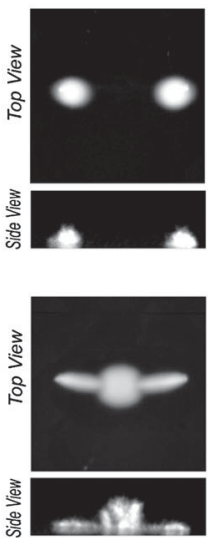
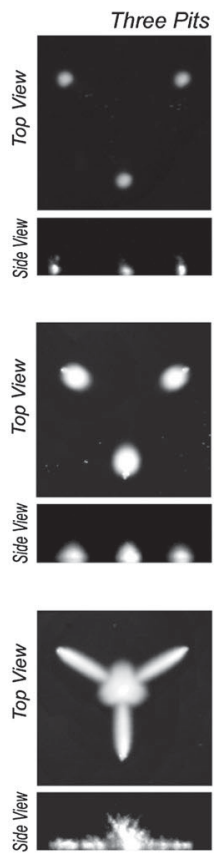

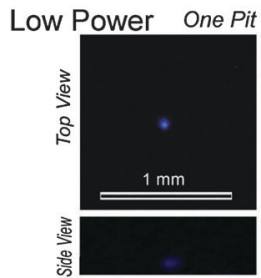

\section{Medium Power}

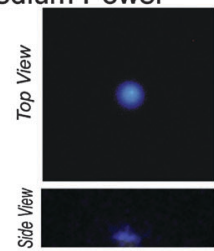

High Power

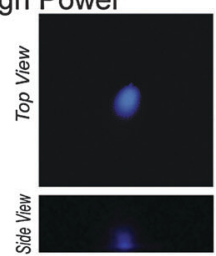

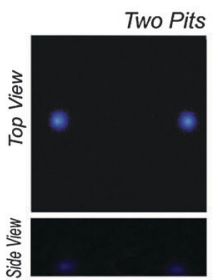
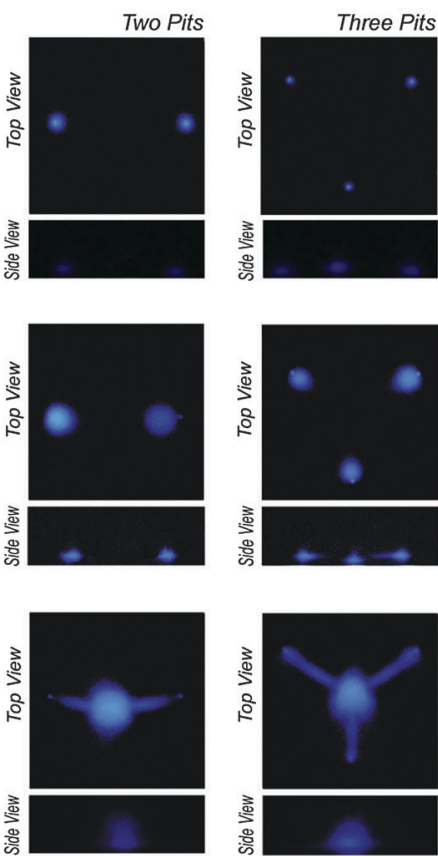

Fig. 14 Bubble patterns generated from one, two and three pit arrangements for increasing acoustic powers: low (0.074 W), medium $(0.182 \mathrm{~W})$, and high $(0.629 \mathrm{~W})$. The left figure is the visible bubble streamers nucleated from the pits. The right figure comprises long exposure photographs under dark conditions of the same bubbles in luminol solution. Reproduced with permission from ref. 79. Copyright $2010 \mathrm{Wiley-VCH} \mathrm{Verlag}$ $\mathrm{GmbH} \& \mathrm{Co}$. KGaA. 


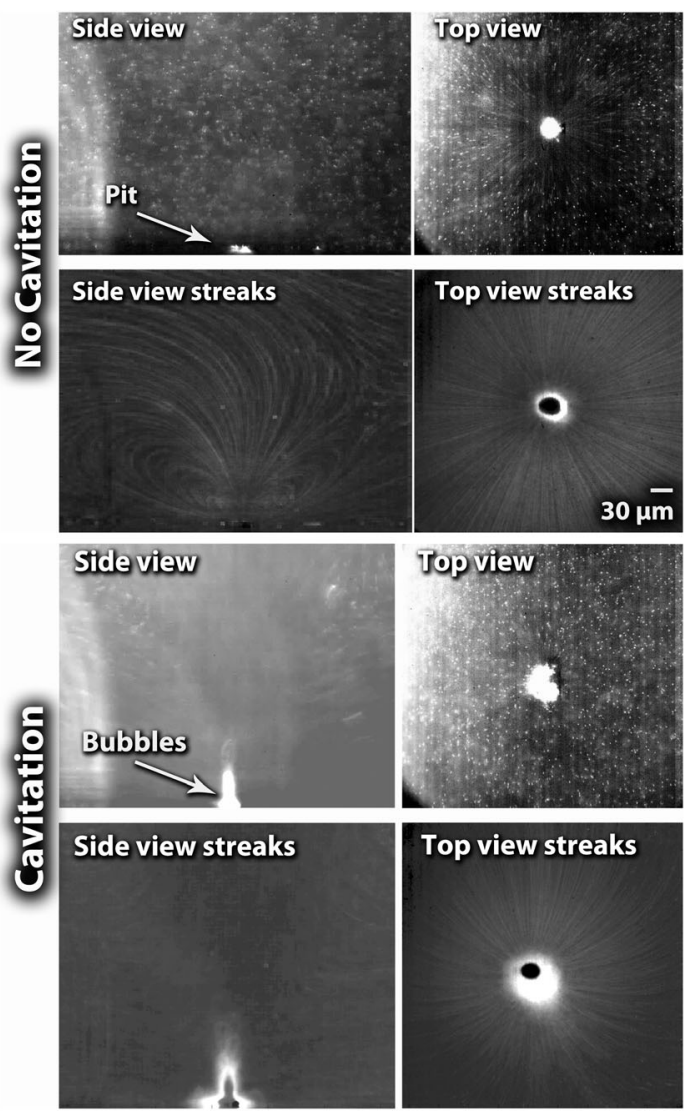

Fig. 15 Streaming effects at $256 \mathrm{kHz}$ induced by the presence of a pit on a silicon surface when there are no bubbles ejected at $70 \mathrm{kPa}$ (left) The streak lines are changed and an increase in the liquid circulation takes place when bubbles are nucleated from the pit at $80 \mathrm{kPa}$ applied pressure (right)

More recent studies based on such a micro-sono-reactor also revealed that SL and SCL (assessed in aqueous luminol solutions) are directly related to the number of pits, which produce bubble clouds and cause a streaming effect. By monitoring SL in water and aqueous propanol, evidence of transient cavitation bubbles could be inferred from the latter. It is also noteworthy that SL and SCL in aqueous solutions gave different light intensities trends, which suggest that bubble populations responsible for light emission are different from the number of chemically-active bubbles. ${ }^{95}$

The control of nucleation sites also allows us to know with certain precission what bubble size distribution exists under given conditions, and a direct relation between the chemical effects can be obtained. This advantage has not only practical uses, but numerical modeling can be brought into play, in order to find optimum operation conditions that normally are not achievable with conventional sono-reactors. Among the other phenomena which can be recorded with such a reproducible and stable system are shockwaves, jetting and surface erosion. $^{96}$

Furthermore, the control of bubble nucleation sites represents an effective tool for the optimization of a given reaction; since different frequencies can be obtained with one system (in contrast to most conventional sono-reactors which operate at a fixed frequency) and different amplitudes provide the desired bubble sizes and numbers. Fig. 16 shows the same micro-sonoreactor system monitoring zones of $\mathrm{OH}^{\bullet}$ radical production in the presence of luminol and SL signal at different frequencies.

Bubble cavitation close to surfaces is sometimes regarded as a negative effect (for instance, erosion of ship propellers). This phenomenon has been extensively used in conventional US cleaning baths. Traditionally when a surface needs to be cleaned, it is completely immersed in a liquid (e.g. water, acetone, ethanol or mixtures thereof) contained in a beaker and then sonicated. The cleaning effect takes place as bubbles nucleate randomly and cavitate close to the target surface. This means that sonication times can be quite long (more than $15 \mathrm{~min}$ ) and there is little control on the cleaning areas and rates. Recently, using the micro-sono-reactor with micromachined pits described above, it was demonstrated that a localized and faster cleaning of surfaces occurs with very small reaction volumes around $200 \mu \mathrm{L} .^{38}$ The removal of metals, polymers and biomaterials (including cells and biofilms) may certainly find applications in surface science and biomedicine.

\section{Modulated}

1 pit
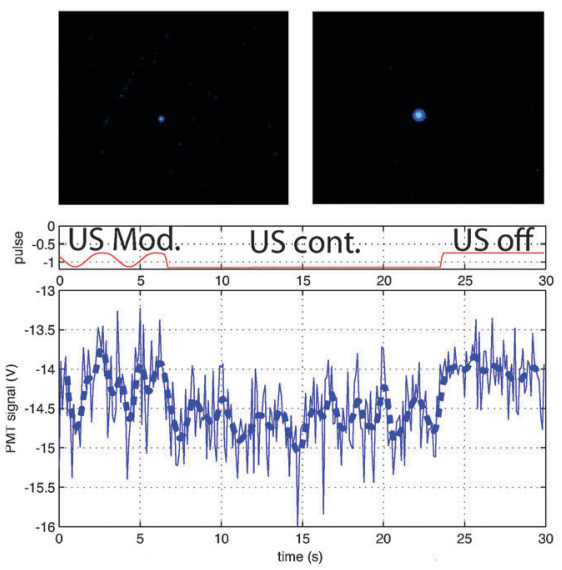

Continuous

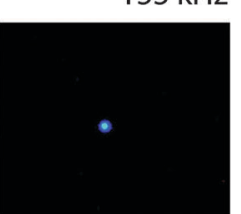

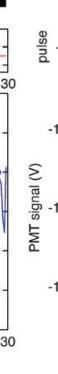

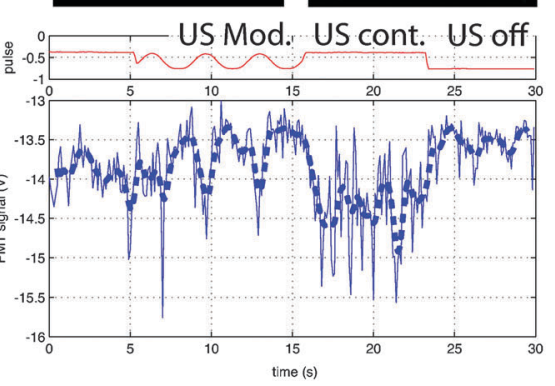

Modulated

1 pit

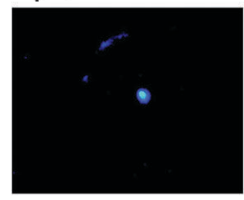

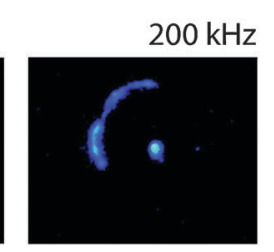

Continuous Modulated

Continuous

$$
1 \text { pit }
$$
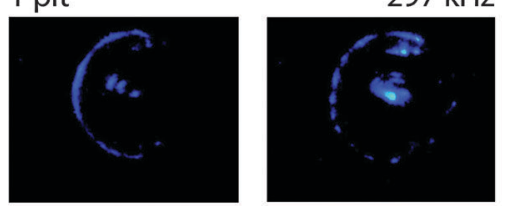

US Mod , US cont.

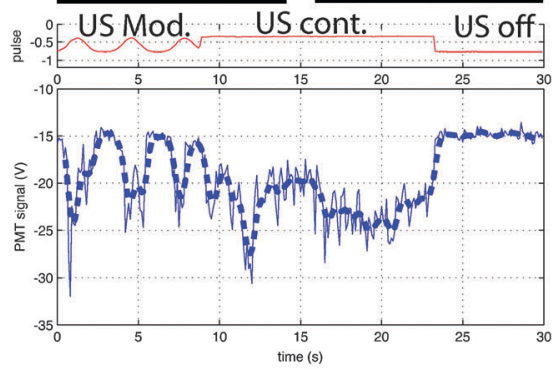

Fig. 16 Top row shows areas of OH radical production via enhanced SCL (sonochemiluminescence of luminol). The bubbles were nucleated from one pit. For comparative purposes, continuous and modulated $(0.3 \mathrm{~Hz})$ oscillations are given for SL signals at the bottom charts. 


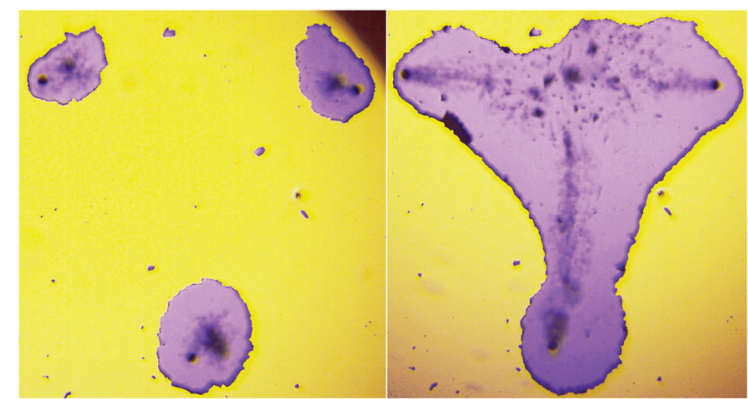

Fig. 17 When a glass slide with deposited material is placed vertically close to three pits, cavitation bubbles emerge as the system is sonicated at low (left) and high power (right), removing gold (yellow) deposited on a glass slide. The pits are separated by a $1 \mathrm{~mm}$ distance. For more details see ref. 38 .

Fig. 17 shows facile removal of gold films deposited on glass slides when subjected to low- and high-power cavitation from three pits. Results were compared with conventional US bath cleaning effects, showing the advantages of small volumes and sonication times needed for the micro-sono-reactor (in less than $1 \mathrm{~min}$ ).

Since micromachined-pitted surfaces can be used to form microbubble clusters capable of producing mechanical effects (microstreaming, shock waves), other insightful mechanochemical reactions achieved recently in conventional sonochemical flasks could likewise be miniaturized. In this context, we highlight ultrasound-aided polymer scission, which is not a random process and takes place preferentially at weakened positions of the chain (mechanophores). ${ }^{37}$ Preliminary experiments have been conducted on ring-closing metathesis (RCM) of diallyl malonate using a Ru-based heterocyclic catalyst ${ }^{97}$ in the same type of micro-sono-reactor with micropits. ${ }^{98}$ As reported by Sijbesma and co-workers, a polymeric catalyst with a molecular weight of $c a .34 \mathrm{kDa} \mathrm{mol}^{-1}$ should feel the forces generated by ultrasound, whereas this mechanical tension is negligible in the case of a control catalyst having small side chains (Scheme 3). ${ }^{99}$

Experiments using similar operation conditions as described above, using one and two pits, have been performed at different frequencies ( $c a .170$ and $230 \mathrm{kHz}$ ). Using the same concentration of reactants (malonate: $200 \mathrm{mM}$; catalyst: $0.2 \mathrm{mM}$ ), cavitation from the micromachined system (one pit, $0.04-005 \mathrm{~W}, V=0.5 \mathrm{~mL}$ )

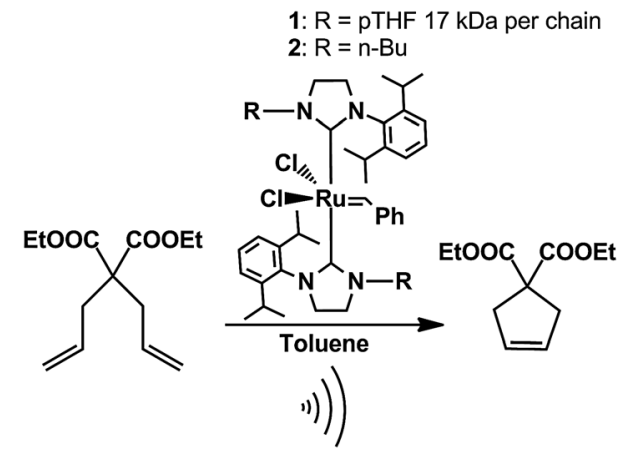

Scheme 3 Ring-closing metathesis reaction conducted with a polymer-based catalyst, which undergoes selective breaking under sonication. gave a conversion of only $1.14 \%$ after 30 min sonication; which contrasts with the conventional sono-reactor (horn: $10-15 \mathrm{~W}, V=5 \mathrm{~mL}$ ) yielding $c a .30 \%$-product within the same time. Clearly this batch micro-sono-reactor needs to be improved, although greener approaches have already been formulated in microreactor technology as scaling-up is not usually required. ${ }^{100}$ The latter could be accomplished either by increasing the number of pits or using a continuous protocol ${ }^{101}$ (a 30 times recirculation would probably afford the conventional $30 \%$-conversion). Finally, and bearing in mind the above-mentioned results with micromachined silicon, it should not be difficult to find the optimal frequency-pressure conditions to increase efficiency, a difficult task with conventional sono-reators.

\section{Conclusions}

Microreactor technology has become a valuable and convenient approach to fulfil the criteria of green chemistry. While microfluidics was initially confined to analytical methods such as HPLC or capillary electrophoresis, miniaturization of continuous-flow reactors offers a series of inherent pluses in chemical synthesis, high-throughput analysis, and the development of sensors that can be tailored for multiple applications. One cannot of course apply microfluidics to every conceivable chemical scenario, and numerous hurdles need to be considered. In order to improve both mass and energy transfer reactions, and to avoid formation of solid by-products, microreactors can now be equiped with piezoelectric transducers of miniature size as well. This innovative area is still in its infancy, though it should afford a new generation of lab-onchip devices. The resulting micro-sono-reactors are capable of managing small reaction volumes in a reproducible and efficient way, while other parameters such as acoustic power, frequency, and sonication times can be fine-tuned. In addition, processes are scalable and enable flow recirculation. Last, but not least, sonochemical reactions conducted on micro-sonoreactors prove that there is a considerable enhancement of radical production and sonoluminescence, which can be harnessed in synthetic transformations in the near future.

\section{Acknowledgements}

The authors would like to express their gratitude to A. G. Zijlstra for supplying particle flow videos and B. Verhagen with assistance in the streak images processing. R. Groote and R. T. M. Jakobs are acknowledged for their collaboration with RCM experiments. This research was supported by the Technology Foundation STW, Applied Science Division of NWO and the Technology Programme of the Ministry of Economic Affairs, The Netherlands.

\section{References}

1 K. Geyer, J. D. C. Codée and P. H. Seeberger, Chem.-Eur. J., 2006, 12, 8434-8442.

2 G. M. Whitesides, Nature, 2006, 442, 368-373.

3 J. M. Ottino and S. Wiggins, Philos. Trans. R. Soc. London., Ser. A, 2004, 362, 923-935.

4 D. Chiu, Anal. Bioanal. Chem., 2007, 387, 17-20. 
5 D. Mark, S. Haeberle, G. Roth, F. von Stetten and R. Zengerle, Chem. Soc. Rev., 2010, 39, 1153-1182.

6 R. Feynman, Eng. Sci., 1960, 23(5), 22-36.

7 J.-C. Charpentier, Chem. Eng. J., 2005, 107, 3-17.

8 A. Thayer, Chem. Eng. News, 2005, 83(22), 43-52.

9 P. S. Dittrich and A. Manz, Nat. Rev. Drug Discovery, 2006, 5, 210-218.

10 A. Bennett, Filtr. Sep., 2010, 47, 16-19.

11 P. L. Mills, D. J. Quiram and J. F. Ryley, Chem. Eng. Sci., 2007, 62, 6992-7010

12 W. Ehrfeld, V. Hessel and H. Löwe, Microreactors: New Technology for Modern Chemistry, Wiley-VCH Verlag GmbH \& Co. KGaA, 2000, ch. 1, pp. 5-8.

13 T. M. Squires and S. R. Quake, Rev. Mod. Phys., 2005, 77, 977-1026.

14 J. Edel and A. De Mello, Nanofluidics: Nanoscience and Nanotechnology, Royal Society of Chemistry, 2009, ch. 3, pp. 47-63.

15 D. Fernandez Rivas, Rev. Cubana Fis., 2008, 25, 142-149.

16 D. Fernandez Rivas, Rev. Cubana Fis., 2011, 28, p. 60.

17 D. Di Carlo, Lab Chip, 2009, 9, 3038-3046.

18 J. G. Santiago, Anal. Chem., 2001, 73, 2353-2365.

19 H. A. Stone and S. Kim, AIChE J., 2001, 47, 1250-1254.

20 K. S. Suslick and G. J. Price, Annu. Rev. Mater. Sci., 1999, 29, 295-326.

21 T. G. Leighton, Prog. Biophys. Mol. Biol., 2007, 93, 3-83.

22 P. Cintas, S. Mantegna, E. C. Gaudino and G. Cravotto, Ultrason. Sonochem., 2010, 17, 985-989.

23 G. Cravotto, W. Bonrath, S. Tagliapietra, C. Speranza, E. C. Gaudino and A. Barge, Chem. Eng. Process., 2010, 49, 930-935.

24 K. Suslick, S. Doktycz and E. Flint, Ultrasonics, 1990, 28, 280-290.

25 T. J. Mason and J. P. Lorimer, Applied Sonochemistry. The Uses of Power Ultrasound in Chemistry and Processing, Wiley-VCH, Weinheim, 2002.

26 C. Leonelli and T. J. Mason, Chem. Eng. Process., 2010, 49, 885-900.

27 J. H. Bang and K. S. Suslick, Adv. Mater., 2010, 22, 1039-1059.

28 K. S. Suslick, D. A. Hammerton and R. E. Cline, J. Am. Chem. Soc., 1986, 108, 5641-5642.

29 E. J. Hart, C. H. Fischer and A. Henglein, J. Phys. Chem., 1990, 94, 284-290.

30 G. Cravotto and P. Cintas, Chem. Soc. Rev., 2006, 35, 180-196.

31 M. Margulis, Russ. J. Phys. Chem., 2006, 80, 1698-1702.

32 K. S. Suslick, N. C. Eddingsaas, D. J. Flannigan, S. D. Hopkins and H. Xu, Ultrason. Sonochem., 2011, 18, 842-846.

33 H. Xu and K. S. Suslick, Phys. Rev. Lett., 2010, 104, 244301.

34 M. M. Caruso, D. A. Davis, Q. Shen, S. A. Odom, N. R. Sottos, S. R. White and J. S. Moore, Chem. Rev., 2009, 109, 5755-5798.

35 G. Cravotto and P. Cintas, Chem. Soc. Rev., 2009, 38, 2684-2697.

36 G. Cravotto and P. Cintas, Chem.-Eur. J., 2010, 16, 5246-5259.

37 G. Cravotto and P. Cintas, Chem. Sci., 2012, 3, 295-307.

38 D. Fernandez Rivas, B. Verhaagen, J. R. T. Seddon, A. G. Zijlstra, L.-M. Jiang, L. W. M. van der Sluis, M. Versluis, D. Lohse and H. J. G. E. Gardeniers, Biomicrofluidics, 2012, 6, 034114.

39 R. Karshafian, P. D. Bevan, R. Williams, S. Samac and P. N. Burns, Ultrasound Med. Biol., 2009, 35, 847-860.

40 P. Riesz and T. Kondo, Free Radicals Biol. Med., 1992, 13, 247-270.

41 B. Ahmed-Omer, D. Barrow and T. Wirth, Chem. Eng. J., 2008, 135(Supplement 1), S280-S283.

42 K. Suslick, Y. Didenko, M. Fang, T. Hyeon, K. Kolbeck, W. McNamara, M. Mdleleni and M. Wong, Philos. Trans. R. Soc. London, Ser. A, 1999, 357, 335.

43 S. Freitas, G. Hielscher, H. P. Merkle and B. Gander, Ultrason. Sonochem., 2006, 13, 76-85.

44 J. Friend and L. Y. Yeo, Rev. Mod. Phys., 2011, 83, 647-704.

45 C. A. Speed, Rheumatology, 2001, 40, 1331-1336.

46 B. Dollet, S. M. van der Meer, V. Garbin, N. de Jong, D. Lohse and M. Versluis, Ultrasound Med. Biol., 2008, 34, 1465-1473.

47 S. Song, Z. Shen, L. Chen, A. A. Brayman and C. H. Miao, Gene Ther., 2011, 18, 1006-1014.

48 T. Franke, A. R. Abate, D. A. Weitz and A. Wixforth, Lab Chip, $2009,9,2625-2627$.
49 Z. Wang and J. Zhe, Lab Chip, 2011, 11, 1280-1285.

50 K. Kulkarni, J. Friend, L. Yeo and P. Perlmutter, Lab Chip, 2009, 9, 754-755.

51 J. Sedelmeier, S. V. Ley, I. R. Baxendale and M. Baumann, Org. Lett., 2010, 12, 3618-3621.

52 R. L. Hartman, J. R. Naber, N. Zaborenko, S. L. Buchwald and K. F. Jensen, Org. Process Res. Dev., 2010, 14, 1347-1357.

53 T. Noel, J. R. Naber, R. L. Hartman, J. P. McMullen, K. F. Jensen and S. L. Buchwald, Chem. Sci., 2011, 2, 287-290.

54 R. L. Hartman, Org. Process Res. Dev., 2012, 16, 870-887.

55 T. Horie, M. Sumino, T. Tanaka, Y. Matsushita, T. Ichimura and J.-i. Yoshida, Org. Process Res. Dev., 2010, 14, 405-410.

56 S. Aljbour, H. Yamada and T. Tagawa, Chem. Eng. Process., 2009, 48, 1167-1172.

57 S. Hübner, S. Kressirer, D. Kralisch, C. Bludszuweit-Philipp, K. Lukow, I. Jänich, A. Schilling, H. Hieronymus, C. Liebner and K. Jähnisch, ChemSusChem, 2012, 5, 279-288.

58 J. Rooze, E. V. Rebrov, J. C. Schouten and J. T. Keurentjes, Ultrason. Sonochem., 2011, 18, 209-215.

59 M. Plesset and A. Prosperetti, Annu. Rev. Fluid Mech., 1977, 9, 145-185.

60 A. Prosperetti, Ultrasonics, 1984, 22, 69-77.

61 T. J. Mason, Practical Sonochemistry, A User's Guide to Applications in Chemistry and Chemical Engineering, Ellis Horwood Publishers, Chichester, 1991.

62 T. Laurell, F. Petersson and A. Nilsson, Chem. Soc. Rev., 2007, 36, 492-506.

63 F. Petersson, A. Nilsson, C. Holm, H. Jonsson and T. Laurell, Lab Chip, 2005, 5, 20-22.

64 J. Nilsson, M. Evander, B. Hammarström and T. Laurell, Anal. Chim. Acta, 2009, 649, 141-157.

65 G. G. Yaralioglu, I. O. Wygant, T. C. Marentis and B. T. KhuriYakub, Anal. Chem., 2004, 76, 3694-3698.

66 F. Petersson, L. Åberg, A.-M. Swård-Nilsson and T. Laurell, Anal. Chem., 2007, 79, 5117-5123.

67 R. Liu, J. Yang, M. Z. Pindera, M. Athavale and P. Grodzinski, Lab Chip, 2002, 2, 151-157.

68 P. Marmottant, J. Raven, H. Gardeniers, J. Bomer and S. Hilgenfeldt, J. Fluid Mech., 2006, 568, 109-118.

69 D. Ahmed, X. Mao, J. Shi, B. K. Juluri and T. J. Huang, Lab Chip, 2009, 9, 2738-2741.

70 P. Rogers and A. Neild, Lab Chip, 2011, 11, 3710-3715.

71 Y. Iida, T. Tuziuti, K. Yasui, A. Towata and T. Kozuka, Ultrason. Sonochem., 2007, 14, 621-626.

72 J. Lee, T. Tuziuti, K. Yasui, S. Kentish, F. Grieser, M. Ashokkumar and Y. Iida, J. Phys. Chem. C, 2007, 111, 19015-19023.

73 Tandiono, S.-W. Ohl, D. S.-W. Ow, E. Klaseboer, V. V. T. Wong, A. Camattari and C.-D. Ohl, Lab Chip, 2010, 10, $1848-1855$

74 S. Kuhn, T. Noël, L. Gu, P. L. Heider and K. F. Jensen, Lab Chip, 2011, 11, 2488-2492.

75 J.-L. Luche, Synthetic Organic Sonochemistry, Plenum Press, New York, 1998.

76 K. Yasuda, T. Torii, K. Yasui, Y. Iida, T. Tuziuti, M. Nakamura and Y. Asakura, Ultrason. Sonochem., 2007, 14, 699-704.

77 M. Ashokkumar, D. Sunartio, S. Kentish, R. Mawson, L. Simons, K. Vilkhu and C. Versteeg, Innovative Food Sci. Emerging Technol., 2008, 9, 155-160.

78 T. Tuziuti, K. Yasui, T. Kozuka, A. Towata and Y. Iida, J. Phys. Chem. A, 2006, 110, 10720-10724.

79 D. Fernandez Rivas, A. Prosperetti, A. G. Zijlstra, D. Lohse and H. J. G. E. Gardeniers, Angew. Chem., Int. Ed., 2010, 49, 9699-9701.

80 D. Flannigan and K. Suslick, Nature, 2005, 434, 52-55.

81 R. Pflieger, H. Brau and S. Nikitenko, Chem.-Eur. J., 2010, 16, 11801-11803.

82 Y. T. Didenko and K. S. Suslick, Nature, 2002, 418, 394-397.

83 S. Hatanaka, H. Mitome, K. Yasui and S. Hayashi, J. Am. Chem. Soc., 2002, 124, 10250-10251.

84 M. P. Brenner, S. Hilgenfeldt and D. Lohse, Rev. Mod. Phys., 2002, 74, 425-484.

85 D. Lohse, Nature, 2005, 434, 33-34.

86 M. Ashokkumar, Ultrason. Sonochem., 2011, 18, 864-872. 
87 M. Ashokkumar, Proceedings of the Meeting of the Japan Society of Sonochemistry (JSS) and The International Workshop on Advanced Sonochemistry. November, Nagoya, Japan, 2011, p. S3.

88 G. Andaluri, E. V. Rokhina and R. P. Suri, Ultrason. Sonochem., 2012, 19, 953-958.

89 N. Golash and P. R. Gogate, Ultrason. Sonochem., 2012, 19, 1051-1060.

90 Y. Son, M. Lim, J. Khim and M. Ashokkumar, Ultrason. Sonochem., 2012, 19, 16-21.

91 Y. Iida, K. Yasui, T. Tuziuti, M. Sivakumar and Y. Endo, Chem. Commun., 2004, 2280-2281.

92 A. Coleman, T. Kodama, M. Choi, T. Adams and J. Saunders, Ultrasound Med. Biol., 1995, 21, 405-417.

93 Tandiono, S. Ohl, D. Ow, E. Klaseboer, V. Wong, R. Dumke and C. Ohl, Proc. Natl. Acad. Sci. U. S. A., 2011, 108, 5996-5998.

94 N. Bremond, M. Arora, C. D. Ohl and D. Lohse, Phys. Rev. Lett., 2006, 96, 224501.
95 D. Fernandez Rivas, M. Ashokkumar, T. Leong, K. Yasui, T. Tuziuti, S. Kentish, D. Lohse and H. J. G. E. Gardeniers, Ultrason. Sonochem., 2012, 19, 1252-1259.

96 D. Fernandez Rivas, L. Stricker, A. G. Zijlstra, H. J. G. E. Gardeniers, D. Lohse and A. Prosperetti, Ultrason. Sonochem, 2012, DOI: $10.1016 /$ j.ultsonch.2012.07.024, in press.

97 R. Groote, B. M. Szyja, E. A. Pidko, E. J. M. Hensen and R. P. Sijbesma, Macromolecules, 2011, 44, 9187-9195.

98 D. Fernandez Rivas, R. Groote and R. Jakobs, unpublished results.

99 A. Piermattei, S. Karthikeyan and R. P. Sijbesma, Nat. Chem., 2009, 1, 133-137.

100 B. P. Mason, K. E. Price, J. L. Steinbacher, A. R. Bogdan and D. T. McQuade, ChemInf., 2007, 38(36), DOI: 10.1002/ chin. 200736259 .

101 N. G. Anderson, Org. Process Res. Dev., 2012, 16, 852-869.

102 E. Ciawi, J. Rae, M. Ashokkumar and F. Grieser, J. Phys. Chem. B, 2006, 110, 13656-13660. 